\title{
Analysis and Implementation of Semaphore Signalling in Railway Tracks
}

\author{
K. Sri Dhivya Krishnan ${ }^{1}$, M. Barathi Selvaraj ${ }^{2}$, P. Rekha ${ }^{2}$, S. Gowtham ${ }^{2}$ \\ ${ }^{1}$ Embedded System Technologies, Knowledge Institute of Technology, Anna University, Chennai, India \\ ${ }^{2}$ Faculty of Electrical and Electronics Engineering, Knowledge Institute of Technology, Salem, India
}

\section{Email address:}

sridhivya.sridhivya@gmail.com (K. Sri Dhivya Krishnan), bseee@kiot.ac.in (M. Barathi Selvaraj), rekhakala3035@gmail.com (P. Rekha), sgeee@kiot.ac.in (S. Gowtham)

\section{To cite this article:}

K. Sri Dhivya Krishnan, M. Barathi Selvaraj, P. Rekha, S. Gowtham. Analysis and Implementation of Semaphore Signalling in Railway Tracks. International Journal of Science, Technology and Society. Vol. 3, No. 2, 2015, pp. 65-68. doi: 10.11648/j.ijsts.20150302.15

\begin{abstract}
Railway is the most economist mode for transport in the world. The signaling in the railways plays the vital role to direct the trains in a right way at right speed towards the destination. In railways a single miscommunication in the signaling may lead to catastrophe. This design makes an approach to improve the mode of communication (signalling) using ARM 8 processor and to avoid the train collision using semaphore signaling. The location of the train is detected at various parts of same track and it transfers through GSM technology with the control of ARM Processor When two trains travel in same track the current location of the train is sent to the control station. GSM network transfer the data immediately to the control station from the station, alert signal is fed to the pilot and the collision can be avoided. On the other hand the warning signal and controlling signals to the train can also be transferred to the loco pilot using Power Line Carrier Communication (PLCC).Thus PLCC avoids signal cables nexus.
\end{abstract}

Keywords: ARM Processor, Semaphore, PLCC (Power Line Carrier Communication), GSM, Track sensors

\section{Introduction}

Today train is the most widely used transport system but accidents are occurring due to collision and signal failure. The goal of the system is to improve the mode of the communication (signaling), which is successfully avoiding the train collision using semaphore signaling and Power Line Carrier Communication (PLCC). Train can collided due to human error and usage of faulty equipment.

To avoid the train collision, in case of two trains approaching in the same track at the same time oppositely with high speed which leads to collision. PLCC technology uses advanced modulation techniques which imposes the data signal of high frequency over the low frequency power signal. The system ensures the reliability and accuracy of the train location using sensor modules.

RTOS provides for the use of semaphores for signaling, when the first train is start on the track, a signal is set as high priority (1) and the signal for the other train has low priority (0).

\subsection{Literature Survey}

In Ji chan Maeng et $a l^{[1]}$ describes the APIs (Application Programming Interface) that can capture of typical RTOS services for specific application. When multiple tasks are running and it share the same resource with an interrupt programming it blocks the signals and executes the priority basis high priority tasks executes at first through the ISR service. Semaphore pattern, which is subject to transforms rules. It handles some APIs that access shared resources are often accessed by several concurrent threads. The implementation is developed by automated tool transPI specific tool performs integrated model based set.

Xi Fang, et al ${ }^{[2]}$ 'Smart Grid - The New and Improved Power Grid: A Survey 'Power Line Communications (PLC): PLC is a technology for carrying data on a conductor also used for electric power transmission. It is used for remote metering and load control applications. PLC in Smart Grid (SG) is still research one. The SG could use many different communications technologies, PLC is the wired technology that has deployment cost comparable to wireless technologies. Technically, in PLC power electronics are used to manipulate high-voltage waveforms for signal and information oriented 
applications.

In Ramesh.et al ${ }^{[3]}$ designed system with the case with all modes of high-speed travel of train there are different rail conditions (wear, corrosion etc.), there are a significant number of potential defects possible and the task has to be performed with some speed. This analysis is detecting the cracks in the track. These deficiencies are not controlled at early stages they might cause huge economic problems affecting the rail network (unexpected requisition of spare parts, handling of incident and/or accidents). The main part of the work was to carry out a feasibility study on two methods for detection of cracks and Avoidance of the collision between the rails. The detection of cracks can be quickly done by ultrasonic waves or sensor.

In Vishnu Krishnan et $a l^{[4]}$ proposes a cost effective solution to the problem of railway track crack detection utilizing Zigbee control assembly which tracks the exact location of track damage which then mended immediately so that many lives will be saved. Train Collision Avoidance System (TCAS) and Anti-collision Device (ACD) are used in this system. The sensor network is a wireless network formed by a group of sensors deployed in same region, which can be used to measure the air pressure, temperature, acceleration, etc. Sensors can transmit signals via radio signal.

In Kiruthiga.M et $a l^{[5]}$ explains in this project, it is proposed to develope automatic railway gate operation to prevent accidents at unmanned gate and automatic closure of unmanned gate. It is also developed to prevent collision of trains running on same track. Automatic closure of unmanned gate reduces the time for which the gate is being kept closed and provides safety to the road users by reducing the accidents. The collision of trains running on same track is also prevented by employing IR Transmitter-Receiver system at each sections of the station and passes the information to a master control room via Zigbee / GSM MODEM.

\subsection{Existing Anti-Collision Device (ACD)}

The ACD system take inputs from GPS satellite system for position updates and network among themselves for exchanging information using their data radio modems and then it takes decision. The system works based on infra-red waves. it consists of an emitter, sensor module and reflector, an emitter and sensor module installed on one train and the reflector is installed on the opposite train. Sensor module senses the presence of train \& the reflector reflects the infrared waves back to the sensor and it activates the alarm.GPS signals sometimes will not perform accurate due to obstacles and network problem. We can overcome this problem in proposed system.

\subsection{Proposed Architecture}

The proposed system is designed in such a way to overcome the difficulties discussed above. The sensors are fixed before the traffic signaling point at a specified distance before and after the station. The sensor sends the data about the location of the trains to control. The data's obtained are analyzed depending upon the distance of the train which is nearest to the station is given first priority. This system works depending upon the priority of trains nearest to the station .This system includes Sensor module. Signal center, Control section and pilot area. Sensor module finds the location of the train.

The traffic signal is opened for the nearest train and train occupies the track in the station and the signal for the train which is waiting is opened. At that time the traffic signal is closed for the opposite train. An alert SMS is given to the pilot who is waiting for signal regarding the availability of the track in the station.

Once the signal is blocked for the train in the station, the signal for the train which is opened. Now the next train can used the regular track to travel. Now both the trains can occupy the tracks that are provided in the station. When the train enters the track the RTOS (Real Time Operating System) detects that event as 1 and the semaphore is an active state until the next train is in waiting state (i.e.) event as 0 .

RTOS supervises inter process communication among the stations so it takes hundreds of CPU instructions to execute the task to avoid collision. In this communications semaphore operations are

- semBCreate() create binary semaphore

- semMCreate() create mutual-exclusion semaphore

- semCCreate() create counting semaphore

- semTake() take semaphore

- semGive() release semaphore

- semDelete() deletes semaphore

\section{Block diagram}

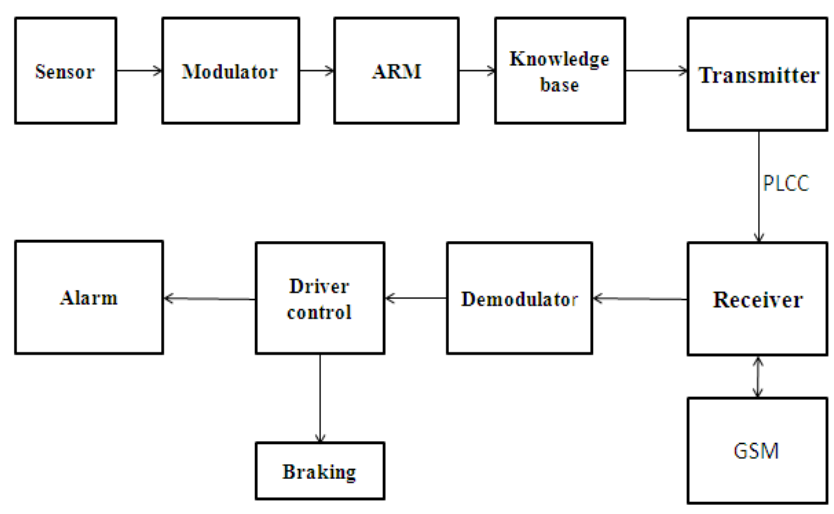

Fig 1. Block diagram of proposed system

\subsection{Hardware Implementation}

The proposed system was designed by using ARM processor and all sub components controlled through the ARM Processor. Power line carrier communication transmit the signal at high voltage stage also which has coupling capacitor to overcome the damage of power lines and its frequency range is $100 \mathrm{~Hz}$ to $50 \mathrm{KHz}$. Coupling capacitor offers the high impedance to power frequency\& low to carrier frequency and attenuation is not much while transmitting which is offers by thicker cross section. In this 
PLCC cross talks practically avoided and noise level is reduced and for network extra lines can be avoided.

ARM 8 Processor: The ARM 8 Cortex processor, it has the ability to scale in speed from $600 \mathrm{MHz}$ to greater than $1 \mathrm{GHz}$. The

Cortex-A8 processor can meet the requirements for poweroptimized mobile devices needing operation in less than $300 \mathrm{~mW}$ and performance-optimized consumer applications requiring 2000 Dhrystone MIPS.The Cortex-A8 highperformance processor because the speed of this processor is high which is mainly used in automotive applications.

\subsection{GSM}

GSM network operate in a number of different carrier frequency ranges which is separated into GSM frequency ranges $2 \mathrm{G}$ and $3 \mathrm{G}$, with most $2 \mathrm{G}$ GSM networks operating in the $900 \mathrm{MHz}$ or $1800 \mathrm{MHz}$. These band limits are already allocated, instead of this the $400 \& 450 \mathrm{MHz}$ frequency. It is designed with moderate level of security. In this system GSM performs transfer the information to the control station and also to the loco pilot.

\section{Software Implementation}

\subsection{Algorithm}

Step1: start the program

Step2: if the track is free set as high priority 1 the train can move on.

Step 3: else the track is engaged set as low priority 0 train has waiting state.

Step 4: stop the program.

\subsection{Flow Chart}



Figure 3.2 Flow chart for semaphore signaling system

\section{Simulation Results}



Figure 2. Block Diagram in LabVIEW 


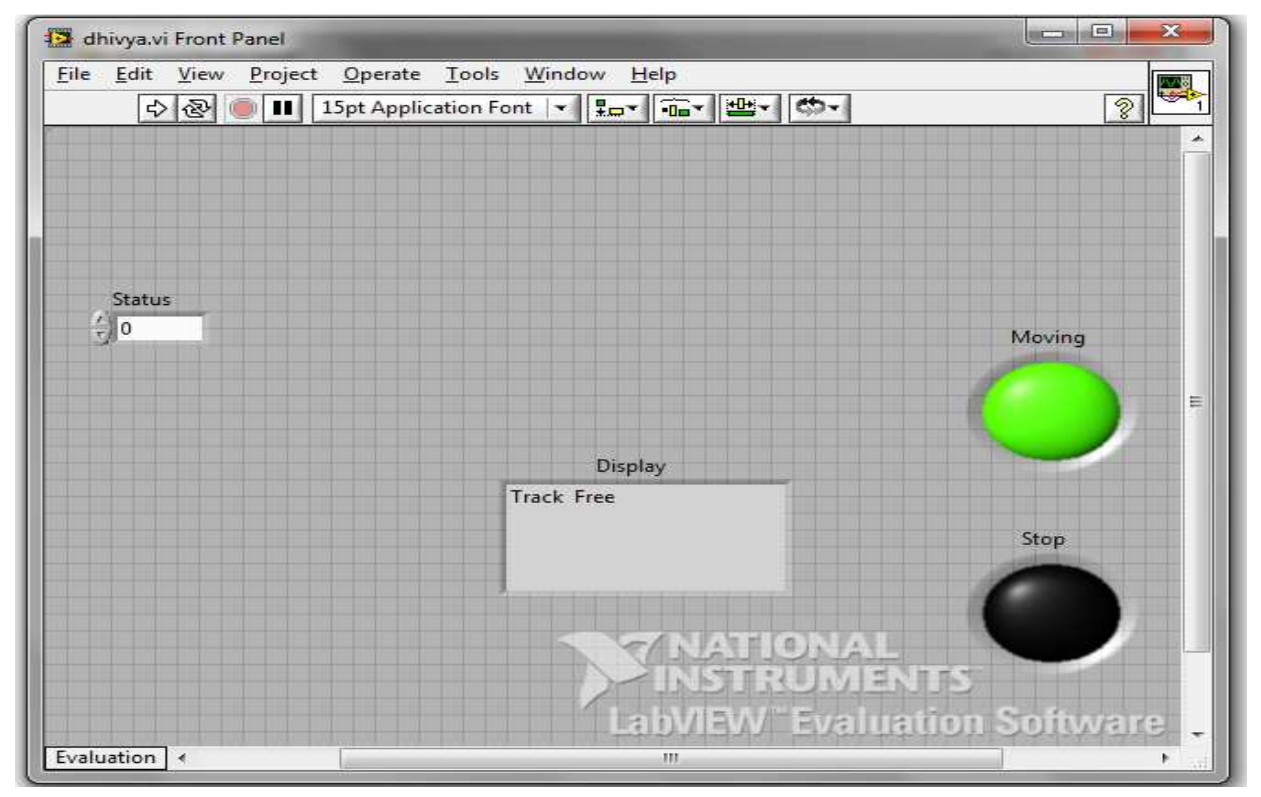

Figure 3. Front panel m LabVIEW

The station 1 and station 2 status are displayed in LabVIEW components. From various signal received from the sensors. The information will be providing to control room. Here it indicates that station 1 is engaged with train and information on the other station also provide at same time. Based on the status of the track (Engaged or free). The train will make its schedule.

\section{Conclusion}

The Proposed system of using semaphore signal technology in railway manages various control signals through PLCC. It is used deluge number of sensors on the long track which senses the status and sends the status to the control station. Thus the received signals are analyzed and resend to the train operator for ease control of train. The signals are transferred through the PLCC which avoids complex cable network connection. In future all the stations are incorporated together using smart automation and intelligent level crossing to avoid train collisions and save the life.

\section{References}

[1] Xi Fang, Smart Grid - The New and Improved Power Grid: A Survey.

[2] B.Vishnukiran and S.Sandeep (2014) A Novel approach railway track damage detection of robot using GPRS Technology. SSRG-IJECE-volume 4.
[3] Elisha.C Mabunda and Cleophas D K Mutephe (2013) Microcontroller Based System Design of Train Collision Avoidance System, IOSR journal of engineering volume 3, issue 2 .

[4] M.Kiruthuga.M, M.Dhivya, P.Dhivya and R.Yugapriya (2014) wireless communication system for railway signal automation at unmanned level, ICETS'14 volume 3.

[5] Malachy Carey and sinead Carvile (2012) scheduling and plat forming trains at busy complex station Transportation research part.

[6] Ji Chan maeng, Jong hyukkim and minsooryu (2006), An RTOS API translator for model driven embedded software development, Real time computing systems and applications.

[7] Simulation of Zigbee based Tacs for Collision Detection and Avoidance for Railway Traffic" Arun.P,Saritha.S, Madhukumar.S, Special Issue of International Journal of Computer Applications on Advanced Computing and Communication Technologies for HPC Applications ACCTHPCA, June 2012.

[8] Train Collision Avoidance Using Vibration Sensor \& MicroController Kishore.P.

[9] Detection of Cracks and Railway Collision Avoidance System, S. Ramesh (IJEEE).

[10] Research on alarm system of railway crossing based On GPS and GPRS", Sudhakar.R. 